\title{
VII.
}

\section{Sogen. Otosclerose bei der Katze}

(Ausgedehnte Spongiosierung des Schläfenbeins obne Ankylose des Steigbügels mit partieller Degeneration des Cortischen Organs und des Nervus cochleae bei einer taub gewordenen Katze).

\author{
Von \\ Professor L. Katz.
}

(Vortrag mit Demonstration, gehalten in der Berl. otologischen Gesellschaft am 13. Februar 1906.)

Mit 2 Abbildungen im Text und Tafel I u. II.

M. H.! Es ist seit meiner ersten Mitteilung im Jahre 1890 uber einen histo-pathologisehen Befund bei der sogenannten Otosklerose eine Reihe sehr wertvoller Arbeiten über denselben Gegenstand ersehienen, welche meinen Befund teils bestätigten, teils ergänzten. Ich möehte in erster Linie erinnern an die älteren, zum Teil sehr eingehenden Studien von Bezold im Jahre 1893, dann von Habermann, Politzer, Siebenmann, E. Hartmann, Scheibe, sodann aber auch an die neueren Beiträge von Panse, Sehwabach, Manasse und Brühl. Aber wenn auch unzweifelhaft durch diese bistologisehen Arbeiten und zwei einschlägige, sehr sorgfältige Monographien von Panse und Denker unser Wissen über diese dunkle und verhängnisvolle Knochenerkrankung gefördert worden ist, so müssen wir doch bekennen, dal wir weder in ätiologisch-therapentischer, noch, was ich ausdrücklieh betone, in anatomisch-pathologischer Hinsicht jetzt schon am Ziele der Erkenntnis dieses Ohrenleidens angelangt sind.

Das, was wir jetzt positiv über die sogenannte Otosklerose wissen, ist ungefähr folgendes: Es handelt sich nicht um eine Schleimhauterkrankung der Pauke, sondern um eine "trockene" Ostitis, die wesentlich auf lakunärer Resorption von festen Knochen und Ersatz durch eine neugebildete, lockere, zum Teil 
hyperostotische Knochenmasse beruht. Es entstehen dadurch neue Markräume und osteoide Substanz, die verschiedene Stadien durchlaufen und finaliter zur Osteoporose resp. Skleroseführen. Hierbei kommt es relativ hänfig zur Stapesankylose.

Das Studium der sogenannten Otosklerose wird noch lange Jahre - darüber kann kein Zweifel sein - im Brennpunkt der otiatrischen Bestrebungen und des otiatrischen Interesses stehen

Fig. 1.

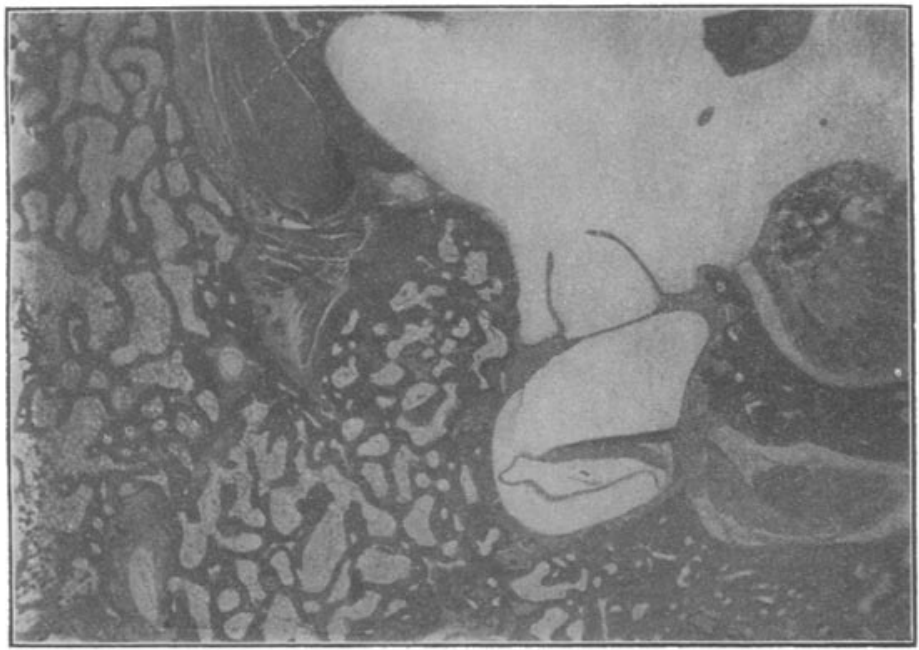

Quersehnitt durch das Pelvis ovalis, den Steigbugel und Vorhof der tauben Katze. Der Knochen ist bis auf eine schmale normale Zone in der Nähe der Stapesplatte uud des Vorhofraumes hochgradig osteoporotisch resp. sclerotisch. Links an der hinteren Wand des Pelvis ovalis eine Hyperostose. Das Ligam. annullare ist ganz intakt. Nerv. vestibul. (rechts) nieht verändert. In dem Photogramm links erstreckt sich die Knochenveränderung bis weit in die hintere Paukenwand.

müssen und wird sicherlich nicht so leicht und so schnell, wie dies in der Otochirurgie zum Segen der Mensehheit geschehen ist, zu so erfreuliehen praktischen Resultaten fübren. Ich halte es deshalb für nicht ganz überflusssig, daß jeder einschlägige Fall, selbst wenn er auf den ersten Blick bedentungslos erscheint, sofern uns nur ein einigermaßen sicheres anatomisehes Substrat zur Verfügung steht, vor Fachgenossen besprochen, resp. demonstriert wird.

Die Gründe, die mich besonders bestimmt haben, diese 
mikroskopischen Präparate einer tauben Katze Ihnen zu zeigen, sind folgende:

1. Es handelt sich hier nicht um einen Menschen, sondern um eines unserer gewöhnlichen Haustiere - um eine Katze -, bei welcher meines Wissens ein derartiger pathologischer Knochenprozeß des Gehörorgans noch nicht beschrieben worden ist.

2. Der pathologische Prozeß ist hier ein sehr alter, ansgedehnter und fast abgelaufener, der sich nicht allein auf die Labyrinthkapsel, sondern auch auf die benachbarten Knochenteile, und speziell auch auf den Hammer und Amboß erstreckt.

Fig. 2.

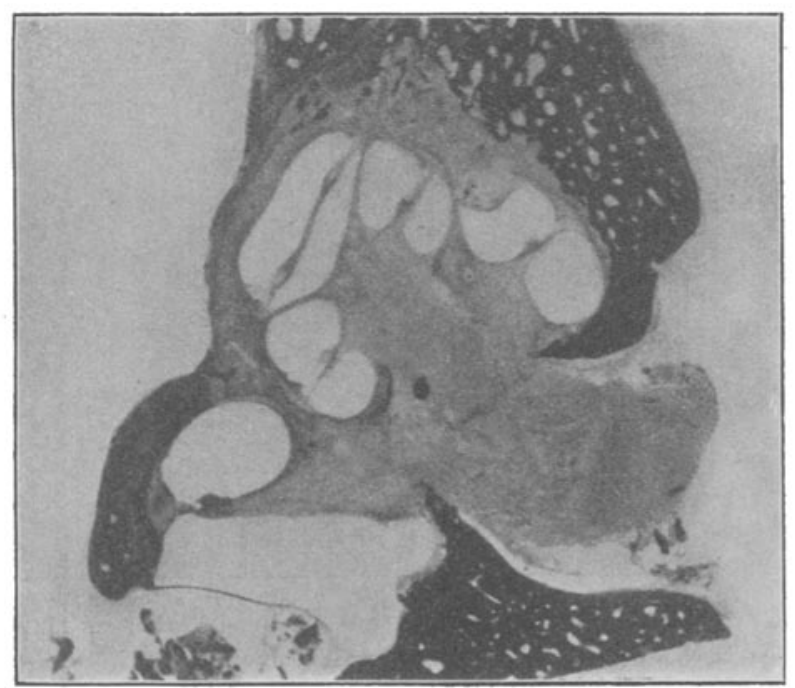

Axialer Sohnitt durch die Schrecke.

Die dunklen Stellen stellen den spongio-sclerotischen Knochen dar. Die äußere Wand der Schnecke (links) ist noch intakt. In den Wandungen des Meatus auditorius internus aasgedehnte Herde von Spongio-Scleresis.

3. Es handelt sich im vorliegenden Fall um eine Kombination von Osteoporose oder Spongiosierung mit deutlicher partieller Degeneration, bezw. Atrophie des Nervus cochleae im Stamm und in den unteren Partien des Modiolus, sowie um Schwund und Degeneration der entsprechenden Ganglienzellen und der Cortischen Zellen (Sinneszellen).

4. Es besteht hier auffallenderweise aber ähnlich, wie in dem von mir im Jahre 1901 im LIII. Band dieses Archivs ver- 
öffentliehten Falle bei ausgedehnter Spongiosierung des Sohläfenbeins ein ganz intaktes Ringband, also keine Ankylose. Bemerken möchte ich beiläufig, daß, wenn wir diese Knochenerkrankung öfter bei Katzen resp. bei Hunden finden werden, was ich nicht bezweifle, oder wenn es uns vielleicht einmal gelingen sollte, diese Knochenerkrankung während des Lebens bei diesen Tieren za diagnostizieren, wir dann die Mögliehkeit erlangen würden, durch experimentelle Untersuchungen auch für die menschliche Otosklerose dabei etwas zu profitieren. Ich glaube aber, daß wir bei dem Charakter der Erkrankung vielmehr von medikamentösen Versuchen, als von chirurgisehen Eingriffen (Stapesextraktion usw.) erwarten dürfen, besonders deshalb, weil ja der patbologische Prozeb mitunter sich, wie Sektionsbefunde beweisen, nicht allein auf das Vorhof-Steigbügelgelenk und seine Umgebung erstreekt, sondern nicht selten andere Gelenke und ziemlich hänfig den Hörnerv in Mitleidensehaft zieht.

Was zunächst die Anamnese dieser Katze betrifft, so bat dieselbe das relativ sehr hohe Alter von 23 Jahren erreicht; sie war dauernd im Besitz von Frau Aubertin, der Mutter des Kollegen A ubertin, des jetzigen Assistenten am physiologisehen Institut, dessen Güte ich den Kopf der Katze verdanke. Es ist sicher festgestellt, daß das Tier in früheren Jahren normal gehört hat, aber in den letzten Jahren stellte sich progressive Schwerhörigkeit ein, die schließlich fast zur vollständigen Taubheit geführt hat. Die Katze litt auch zeitweise an eigentümlichen Erregungszuständen, die auf das Bestehen von subjektiven Geräuschen schließen ließen. Sie erschrak plötzlich, zuckte zusammen und drehte den Kopf herum, als ob sie gernfen worden wäre. Sie hatte ein graues Fell und gelbe Augen. Ioh betone dies deshalb, weil Sie ja wissen, daß unvollkommen albinotische Katzen mit weißem Fell und blauen Augen oft als taub befunden worden sind, und bei denen sich bei der Sektion eine Degeneration und Atrophie des Nervus cochleae und Veränderungen am Cortisehen Organ feststellen ließen. Die Tatsache, daß eine Correlation zwischen albinotischem Zustande und Taubheit besteht, war bereits $B$ uffon im Jahre 1769 und später auch Darwin $u$. a. bekannt, aber zuerst Rawitz und nach ihm in eingehender Weise Alexander, dann Beyer und ich selbst haben durch histologische Untersuchungen dieses Vorkommnis bestätigen können. Ferner erwähne ich, dal die in Rede stehende Katze fast immer im Zimmer gehalten worden ist und niemals geboren 
hatte; das letztere ist zu beachten, weil nicht ganz ohne Grund von einigen Untersuchern der otosklerotische Prozel als Osteo, malacie gedeutet worden ist, welche mit dem Puerperium ja unzweifelhaft nicht selten in ursäoblichem Zusammenhang stehtEndlich sei noch bemerkt, dab die Katze wegen zunehmender Gebrechlichkeit anf Wunsch von Frau Aubertin mit Leuchtgas vergiftet worden ist. - Ioh wende mich nun zu einer kurzen anatomischen Beschreibung.

Aus dem allgemeinen Sektionsbefund möchte ich die folgenden Punkte hervorheben:

1. Im Abdomen fand sich ein ziemlich hühnereigroßer Tumor, der anseheivend rom Uterus ausging und der naoh der mikroskopischen Untersuchung von Herrn v. Hansemann als ein Sarkom festgestellt worden ist.

2. Die Schädeldecke stellte sich beim Durchsägen als verdickt heraus; innerhalb des Gehirns findet sich ein anscheinend gleicher, aber kleinerer Tumor, der mit der Dura mater zusammenhängt nnd vorwiegend im Mittelhirn liegt. Die mikroskopische Untersuchung dieses Tumors, der teilweise verkalkt ist, sonst aber der abdominalen Geschwulst ganz ähnlich sieht, steht noch aus. In der Paukenhöhle ist bei der Inspektion kein Exsudat oder Transsudat zu sehen. Das Trommelfell ist weißblänlich, dunn, die Gehörknöchelchen von normaler Gestalt; der Steigbügel befindet sich in einem etwas verengten Pelvis ovalis; Schleimhäute dünn und blaß, also nichts Pathologisches!

3. Der Nerv im Porus acusticus internus ist makroskopisch nicht verändert.

Was nun aber den mikrosk opis chen Befund des häutigen Labyrinths betrifft, so möchte ich hier von allen unnötỉgen Detailbeschreibungen, die mich zu weit ftihren würden, absehen, und nur das prinzipiell Wichtige in folgenden Daten feststellen:

a) Auf beiden Ohren ist die Labyrinthkapsel und deren knöeherne Umgebung bis weit in die Wandungen des inneren Gehörganges hinein zu mindestens drei Viertel in eine teils osteoporotisehe, teils sklerotische Knochenmasse umgewandelt. Ein einigermaßen normales Knochengewebe findet sich nur in beschränktem Umfange an der vorderen und äußeren Wand der knöchernen Schnecke. Am knöchernen Teil der Pars superior labyrinthi sieht man normalen Knochen nur um die Hohlräume der Bogengänge und des Vorhofes herum. Das kranke Knochen- 
gewebe ist auf dem mikroskopisehen Querschnitt einem Schwamm vergleichbar; die Knochenbälkehen färben sich mit Karmin oder Hämatoxylin distinkt rot oder blau. Sie sind verdickt, haben zum Teil einen lamellären Bau und zeigen entschieden vergrößerte und plumpe Knochenkörperchen (Politzer, E. Hartmann). Höchst selten sieht man noch einige Knorpelzellen in den zurückgebliebenen Interglobularräumen. Innerhalb der so gebildeten Markräume finden sich sehr reichliche Fettzelle $n$ und ein relativ spärliches zartes, kernarmes Bindegewebe mit dazwischen liegenden zartwandigen Blutgefäßen (keine Spur mehr von Riesenzellen oder Osteoblasten). -

b) Das Vorhofs-Steigbügelgelenk ist ganz frei (Fig. 4 auf Taf. I. II). Das Pelvis ovalis zeigt an seinem hinteren Umfang eine Hyperostose von ausgesprochen osteoporotischer Struktur.

e) Hammer-Amboßgelenk mit Meniskus frei (Fig. 1 auf Taf. I. II).

d) Der Querschnitt durch den Hammerkopf und Amboßkörper zeigt bei stärkerer Vergrößerung eine große Reihe größerer und kleinerer, meist runder oder ovaler, durch die Färbung markierter Stellen (Säume), in deren Zentrum man durchschnittene Haversische Kanäle oder Markräume erkennt (Fig. 1 auf Taf. I. II).

e) Diese markant gefärbten Säume bestehen aus neu gebildeten apponierten und später sklerosierten Knochen (Fig. I sd auf Taf. I. II), wie dies beim Menschen schon Siebenmann, Habermann, ich u. a. gesehen haben.

f) Gleichartige kleinere und größere Herde mit zentral gelegenen Löehern finden wir auch im Promontorium, in der Nähe des runden Fensters, sowie vereinzelt anch im Modiolus selbst. Diese Herde stechen durch die intensive Aufnahme des Farbstoffs von ihrer Umgebung stark ab.

g) Das nur in geringem Umfange noeh zuritck gebliebene normale Knochengewebe zeigt auf dem Querschnitt Unebenheiten der Grundsubstanz, ab und zu kleine Risse, und dies besonders an der Grenze des pathologischen, drusenartig nach dem Gesunden begrenzten Knochens. Die Knochenkörperchen des gesunden Knochens sind schwach gefärbt, klein und undeutlich erkennbar.

h) Am Nervus vestibuli und an dem häutigen Gebilde des Vorhofes, speziell am Sacculus und Utriculus ist nichts Pathologisches auffindbar.

i) Dagegen ist im Stamm des Nervus cochleae neben einer 
dentlichen partiellen, interstitiellen Bindegewebswucherung eine deutliche Degeneration und Atrophie zu konstatieren. Diese Atrophie betrifft aber ganz besonders diejenigen Nervenbündel, die zur basalen und zum Teil mittleren Schneekenwindung laufen, resp. dort in die Lamina spiralis ossea eintreten (Fig. 2 Lspo auf Taf. I. II). Hier findet man von eigentlicher Nervensubstanz nur Spuren neben dünnen Bindegewebsfasern. Die dazu gehörigen Ganglienzellen in der unteren, aber aueh in der mittleren Sehneckenwindung sind der Zahl naoh außerordentlich reduziert und auch in der Form verändert (Fig. 2 Gsp auf Taf. I. II). In einigen Quersobnitten des Rosenthalschen Kanals konnte ich nur fünf bis acht Ganglienzellen zählen (Fig. 3 auf Taf. I. II). Besonders deutlich ist diese Atrophie des Nerven bei der Pal-Weigertschen Färbung zu konstatieren.

k) Das Cortische Organ befindet sich an denjenigen Stellen, wo der Nervus cochleae und die Ganglienzellen atrophisch sind, in pathologiseher Schrumpfung, d. b. es ist sehr niedrig, Pfeiler nicht sichtbar (Fig. 2 Co auf Taf. I. II). Aber die abrigen Teile des Ductus cochleae, besonders die Membrana tectoria, Membrana Reissneri, Stria vaseularis befinden sich an normaler Stelle und erseheinen morphologiseh unverändert. Pigmentbefindet sich in spärlicher Menge nur in der Stria vascularis, im Modiolus fast gar nicht.

1) In den oberen Partien der Sehneckenwindung sind dagegen sowohl die Nervenfasern als auch die Ganglienzellen ohne jede nachweisbare Veränderung, speziell am Cortischen Organ sind die zylindrischen Cortischen und die flaschenförmigen Deitersschen Zellen neben normal gebogenen Pfeilern auf das Klarste zu sehen. Das Vas spirale an der unteren Fläche der Membrana basilaris zwischen den Pfeilern ist auffallend weit und in allen Querschnitten sichtbar. Die Gefäße sind sowohl innerhalb des Meatus auditorius internus als auch innerhalb des Modiolus ziemlich dickwandig.

m) SehlieBlich möchte ich noch bemerken, daß die Veränderung des Knochens innerhalb der. Wandungen des Meatus anditorius internus sehr alt und ausgesprochen spongio-skleroti s ch erscheint, ich mőchte glauben, daß hier der erste (älteste) Herd lokalisiert ist.

Ich beto ne ferner, daß die Osteoporose anch in der Squama ossis temporis nachweisbar ist, es befindet sich hier eine sehr schmale Corticalis, weite Markräume mit dünnen Bälkehen und mit Fettzelleninhalt. 
Es entspricht demnach im allgemeinen das pathologisehanatomische Bild des Gehörorgans bei dieser tauben Katze einigermaßen demjenigen, welehes andere Autoren (Bezold, Siebenmann, Habermann, Politzer u. a) und ich selbst in meiner Arbeit im Jahre 1901 im Arehiv, Bd. LIII, bei mensehlicher Spongiosierung beschrieben, und was ich dort in Bezug auf die Art der Entwicklung mit dem Namen Ostitis chronica vaseulosa ossis temporis Volkmann bezeichnet habe. In diesen Fällen war trotz ausgedehnter multipler Spongiosierung manchmal nur eine reeht unerhebliche Beteiligung des Ringbandes zu konstatieren. Ioh verweise hier besonders anf eine Abbildung: von Si ebenman n (Zeitschrift für Ohrenheilkunde, Bd. XXXVI). Im vorliegenden Falle der tauben Katze war aber das Vorhof-Steigbügelgelenk (Ringband), wie Sie es in dem ansgestellten Präparate sehen, durchaus fehlerfrei.

Also, m. H., es kann, wie Sie hier sehen, neben einer ansgedehnten und ausgesprochenen Spongiosierung der Labyrinthwand (sogen. Otosklerose) auch nicht eine Spur von Steigbugelankylose vorhanden sein. Es fragt sich, ob wir nicht wegen dieser, wie gesagt, auch schon beim Menschen gefundenen, von dem Gewöhnlichen abweichenden Lokalisation der Knochenaffektion später werden eine besondere Einteilung des Knochenbefundes vornehmen müssen. Der klinische Begriff der „Otosklerose" deekt sich nicht immer mit dem pathologisch-anatomischen Substrat der "Ankylose des Steigbügels"; und ich glaube bestimmt, daß hier späterhin bei weiteren wissensehaftlichen Fortschritten werden Unterseheidungen gemaeht werden müssen. Eine "Otosklerose" ohne Ankylose des Steigbügels wird m. E. zur Zeit wohl kaum sieher diagnotiziert werden können.

Ich halte es nicht für unwahrscheinlich, daß derjenigen Form der sogenannten Otosklerose, bei welcher gewissermaßen auf den ,ersten Hieb" Steigbuigelankylose auftritt, die deformierende Arthritis zugrunde liegt, während in den nicht ganz seltenen, hauptsäehlich alte Individuen betreffenden Fällen, wo der Prozeb erst spät oder gar nieht die Symphyse ergreift, vielleicht ein osteomalacisches oder syphilitisches Leiden oder sensile Atrophie des Knochens vorliegen können. Bei letzterer kommt Degeneration des Nervus cochleae meiner Erfahrung nach oft gleichzeitig vor. - Unerläßlich wird es deshalb später sein, neben der Untersuchung des Sehläfenbeins anch andere Knochen des Körpers sorgfältig zu prüfen, wenn dies für den Arehiv f. Ohrenheilkonde. LXVIII. Bd. 
Ohrenarzt auch mitunter aus äußeren Gründen schwierig sein dürfte.

Die pathologiseh-anatomische Diagnose dieses Falles würde kurz gefaßt etwa folgendermaßen lauten müssen:

Beiderseitige sehr ausgedehnte alte 0steoporose bezw. Spongiosierung und Sklerosierung mit Hyperostose nicht allein der Labyrinthkapsel, sondern weiterer Partien des Schläfenbeines. Die Herde sind zum groben Teil miteinander versohmolzen, kommen aber anch noch disseminiert vor. Die Gelenke sämtlicher Gehörknöchelchen frei, deutliche partielle Degeneration und Atrophie des Nervus cochleae, sehr starker Sohwund der Ganglienzellen in der unteren, zum Teil auch der mittleren Schneckenwindung. Das Corti sche Organ in der unteren Windung deutlich atrophiseh, dagegen normal in den oberen Abschnitten. Keine Ankylose des Stapes.

Was nun das ätiologische Moment dieser chronischen, durch Resorption und Apposition eharakterisierten Knochenentzindung betrifft, welche als Schlußeffekt zur Osteoporose oder Spongiosierung führt, so bin ich nach wie vor der Überzeugung, dab diesem Prozeb ein k onstitutionelles resp. dyskrasisches, meist ererbtes Leiden zugrunde liegt, anf dessen Grundlage das Schläfenbein bezw. das von ihm amschlossene Ge. hörorgan gewissermaßen eine Art Locus minoris resistentiae für eine Reihe von schädlichen Gelegenheitsursachen darstellt. Wie früher, rechne jch auf Grund meiner Beobachtungen zu diesem konstitutionellen Leiden hauptsächlich die folgenden allgemeinen pathologischen Anlagen:

1. die sog. rheumati sch-gichtische (auf der anatomischen Basis der Ostitis resp. Arthritis deformans),

2. die luetische,

3. die skrofulös-anämische,

4. die neuroparalytische,

zu welch letzterer Anlage man wohl die Altersveränderungen rechnen muß. Alle diese Anlagen disponieren meines Erachtens unter dem Einfluß verschiedenerGelegenheitsursachen, zu denen erfahrungsgemäß vorwiegend Mittelohrkatarrhe verschiedenen Charakters, schlechte Ventilation der Paukenhöhle, Wucherungen im Nasenrachenraum, Nasenaffektionen usw. gehören, zur Entstehung dieser Ostitis.

Den primären Entzündungsherd verlege ich, ebenso wie Habermann, auf Grund histologisoher Beobachtungen mei- 
stens in die mukös-periostale Auskleidung der Paukenhöhle. Von da zieht der ProzeB, wie dies für die Volkmannsche Ostitis chronica vasculosa typiseh ist, schleichend an den GefäBwandungen entlang in den Knochen hinein und bringt durch Bildung von neuen GefäBen und Zellenwucherungen in den Haversischen Kanälen den kompakten Knochen zur Einschmelzung. Dieses erste Stadium zeichnet sich durch das Entstehen von neuen Markräumen aus und zeigt nicht selten neben sehr reichlichen Bildungszellen auch viele Osteoklasten. Späterhin erfolgt stellenweise durch Apposition von neu gebildeten Knochengewebe eine gewisse Einengung der Markräume und Verdichtung (Sklerose) der jungen Knochenbalken. Das Finale des oft ein Mensehenalter währenden Prozesses stellt hänfig die "entzîndliche" Osteoporose dar, wobei wir in den betreffenden, früher neugebildeten Markräumen meist nur Fettgewebe und sehr spärliches kernarmes Bindegewebe mit relativ weiten Blutgefäßen vorfinden. Auf Grund meiner neueren Beobachtungen glaube ich aber, daß der primäre AnstoB zur Labyrinthknochen-Erkrankung auch vom inneren Periost, d. h. von der Dura mater, welche das Schläfenbein bis in den inneren Gehörgang hinein bedeckt, ausgehen kann. Es scheint mir hier dafür die entsprechende Knochenveränderung in meatus auditor. interus zu sprechen.

Ich nehme in diesem Falle einen ganz ähnlichen Vorgang der pathologischen Entwicklung an wie bei der Ostitis resp. Arthritis deformans, bei weleher die häufig zu beobachtenden Verdickungen der Schädelknochen sicher vom in neren Periost ausgehen im Gegensatz zur Rhachitis wo die SchädelknochenVeränderungen nur vom ä $\mathfrak{u}$ Ber en Periost ihren Ausgang nehmen (v. Hansemann). Wir finden aber auch bei der Ostitis deformans neben Verdickungen einerseits inneren Knochenschwund, andrerseits Neubildung von osteoider Substanz.

Sehr interessant sind mir die Beobachtungen und Demonstrationen von v. Hansemann gewesen. Er fand, daß Affen eine außerordentliche Disposition (ererbte Anlage) zur Rhachitis haben; sie bekommen sie aber tatsächlich nur in der Gefangensehaft und zwar durch versehiedene in ihr wirkende Momente, die v. Hansemann "die Domestiziernng" nennt. Ein in seiner Heimat, z. B. in Java, in der Wildnis geschossener Affe zeigt niemals rhachitische Knochenveränderungen; dagegen ist fast jeder längere $7_{\text {eit }}$ sei es hier, sei es in der Heimat, in der Gefangenschaft gehaltene Affe mit Rhachitis anch der Schädel- 
knoehen behaftet. Anch die in Frage stehende taube Katze hat meines Erachtens eine konstitutionelle Anlage zar Knochenerkrankung mit auf die Welt gebracht. Die Gelegenheits-Ursache zum Ausbruch ihrer Osteoporose am Schläfenbein die ich fur das Endstadinm einer Ostisis deformans halte, liegt jedoch wahrscheinlich anch hier in der streng bei ihr durchgeführten langjährigen Domestizierung. Von der gleichzeitig hier bestehenden, meines Erachtens sekundären Nervenatrophie des Nervus cochleae glaube ich, dabi sie zurückgeftihrt werden kann auf die Geschwulsteachexie und den Marasmus dieses sehr alten Tieres. Auch Siebenmann führt in seinem Falle ron Acusticusdegeneration bei Otosklerose das sekundäre Nervenleiden auf Krebseachexie zurück und bezieht sich auf derartige früher veröffentlichte neuropathologische Fälle von 0 ppenheim und Siemerling. Es ist mir selbst aufgefallen, daß längerer Aufenthalt in Irrenhänsem, Gefängnissen und Hospitälern relativ oft bei den betreffenden Insassen durch diese Art von Domestizierung zur Otosklerose bezw. Atrophie des Hörnerven geführt hat. Dab Lues resp. eine lnetische Allgemeinerkrankung leicht Otosklerose veranlassen kann, ist als ganz sicher zu bezeichnen, ich kann aber nicht, ebenso wie dies Denker und andere bereits urgiert haben, Habermann beipfliehten, wenn er die Knochenveränderung bei Otosklerose überhaupt als eine spezifisch syphilitische bezeichnet. Daß die bei der Otosklerose gefundene Periostitis, ferner die Hyperostose, das Vorkommen von multiplen Herden usw. für Lues sprechen können, kann ohne weiteres Habermann zugegeben werden, aber bekanntlich können derartige, oder ähnliche Befunde zum Teil auch bei Knochenerkrankungen anderer Herkunft (z. B. bei Arthritis deformans resp. Ostitis deformans, Osteomalacie ete.) beobachtet werden. Wirkliche Gummiknoten sind bei der Sklerose weder im Knochenmark noch im Periost des Gehörorgans bisher gefunden worden. Ganz sichere Zeichen fur eine bestimmte Klassifizierung des Knochenbefundes fehlen uns zurzeit noch. Gegen das "Spezi"

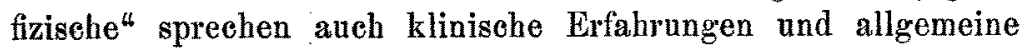
Erwägungen. Daß der Knochen eines Syphilitisch ganz besonders vulnerabel ist und oft selbst durch ein leichtes Tranma zu chronischer Periostitis und Ostitis geführt werden kann, ist durch die Erfahrung bestätigt. In diesem Sinne muß sowohl die ererbte, als anch die später erworbene Syphilis als eine gefährliche Anlage zur Entstehung anch von Otosklerose bezeichnet 
werden. Es gibt bei der sogenannten Otosklerose meines Erachtens aber keine einheitliche spezifizisehe Grundursache od Dyskrasie. Es führen hier viele Wege nach Rom!

Was die Therapie betrifft, so möchte ich nur mit wenigen Worten dieses so undankbare Kapitel berühren. Dis Behandlung ist bekanntermaßen eine wahre Crux furr den Ohrenarzt, die ihm die Beschäftigung mit der Ohrenneilkunde nicht selten sehr verleidet. Vom Standpunkt der Theorie aus hat Siebenmann sicher recht, wenn er mit Phosphor, weleher in der Behandlung der Rhachitis wohl sicher nutzbringend ist, die Otosklerose behandeln will. Auch Politzers Jodkalium, sowie andere Jod enthaltende Präparate können gewiß unter Umständen von Nutzen sein. Hoffentlich wird uns die $\mathrm{Zn-}$ kunft mit Medikamenten bescheren, welche entzündete Knochen günstig beeinflussen können. Von chirurgischen Maßnahmen können wir, wie oben bereits erwähnt, in Hinsicht auf den progredienten Entzindungsprozeb der Erkrankung nicht viel erwarten, und dringend muß vor jeder das Gehörorgan reizenden Therapie, besonders vor intratympanalen Einspritzungen mit nicht indifferenten Flüssigkeiten gewarnt werden, worauf ja bereits früher Lucae und Schwartze aufmerksam gemacht haben. Damit will ich aber nicht die Tatsache leugnen, daß in denjenigen Fällen von Otosklerose, wo der Steigbügel, wie in diesem Falle, noch frei beweglich geblieben ist, ganz ebenso wie bekanutlich bei adhäsiven Mittelohrprozessen durch vorsichtige lokale Behandlung (Lueaes Druoksonde, Pneumomassage, Katheter) nicht selten einige belästigende Symptome, wie subjektive Geräusche, Schwindelgefühl, manchmal gemildert, ja wesentlich gebessert werden können. Die Hauptsache bleibt die rationelle Behandlung der Grundursache, d. h. des dyskrasischen Leidens durch allgemeine teils antirheumatische, teils tonisierende oder kalmierende Kuren und ferner die sorgfältige Vermeidung von allen das Gehör schädigenden Gelegenheitsursachen.

Die Behandlung mit warmen Soolbädern in den entsprechenden Badeorten und den Gebrauch von antigichtisehen Trinkquellen in Wiesbaden, Karlsbad, Salzschlirf usw. halte ich bei der wirklichen Otosklerose für durchaus indiziert. Von inneren Mitteln verabreiche ich für gewöhnlich und öfter mit Erfolg Arsen, Leberthran, eventuell auch Phosphor - in Granulis nach A. Hartmann - aber man muß bei der Giftigkeit des Mittels in der Dosierung recht vorsichtig sein. Für zweckmäßig halte 
ich auch das an organischen Phosphor-Verbindungen reiche "Sanatogen".

Ein besonderes Gewicht möchte ich aber auch auf die rationelle Behandlung etwaiger allgemein-nervöser Störungen legen (Neurasthenie), denn ein Nebeneinanderbestehen von Otosklerose und allgemein-nervösen Zuständen ist doch nicht zu leugnen. Für durchaus falseh halte ich aber das dabei von einigen Nervenärzten ohne Rücksicht auf das Gebörorgan beliebte Kaltwasserverfahren, welches die Besehwerden (besonders Sausen und Schwindel) durchaus verschlimmert. Lauwarme Soolbäder und Aufenthalt im Gebirge sind meiner Erfahrung nach hier relativ am zweekmäßigsten.

\section{Erklärung der Abbildungen auf Tafel I n. II.}

Fig. I. Querschnitt durch den Atticus der Katze, 20 fache Vergrößerung. Färbung Haematoxytin-Eosin.

Ae.: Äuherer Abschnitt des Atticus.

JAtt.: Innerer Abschnitt des Atticus.

A.: Ambos-Körper.

H.: Hammerkopt.

Sel. : Sclerotische Säume, rings un die Markräume oder Haversische Kanale. G.: Gelenkkapsel; das Hammergelenk intakt.

Hb.: Haftband für den kurzen Ambosschenkel.

Fig. II. Axialer Schnitt durch die untere Scbneckenwindung.

G sp.: Canal. spiral. ganglionaris Rosentbalii, in welchem nur sehr wenige Ganglienzellen vorhanden sind und nur einzelne atrophische Nervenfasern. Spärliche Bindegewebsfasern.

L. sp. 0 : Lamina spiralis ossea mit vereinzelten atrophischen Nervenfäserchen und einzelnen Zügen von Bindegewebsfasern.

Co. Cortisches Organ, bis auf ein niedrigen Häufchen von cubischen Zellen verschwunden. - Keine Pfeiler.

RM.: Reissnersche Membran (normal).

Sct. : Scala tympan.

Scl.: Sclerotische Veränderung des Knochens.

Fig. III. Axialer Schnitt durch den Canal spiral. ganglionaris Rosenthalii bei stärkerer Vergrößerung (250). Färbung Haematoxylin-Eosin.

Gz.: Ganglienzellen an $\mathrm{Zahl}$ sehr verringert, in der Form leicht verändert, das Protoplasma zeigt undeutlich Nisslesche Körperchen.

Bg.: Bindegewebsfasern; Nervenfasern sehr spärlich $u$. atrophisch.

Gef.: Gefäß (erweitert).

Lsp.: Lamina spiralis ossea, zwischen ihren zwei Blättern sehr spärliche Nervenfäserchen.

Fig. IV. Querschnitt d. Steigbügel a. Pelvis ovalis. (Färb. mit Haematonexbie).

mt.: muse. tensoris tympani.

La.: Lig. annulare vollständig intakt.

vS.: Vorderer Schenkel des Steigbügels.

HS.: Hinterer Schenkel des Steigbügels.

NK.: Normales Knochengewebe.

Spong.: Spongiosierung resp. Sclerosierung des Knochens.

StP.: Steigbügelplatte.

Hyp.: Hyperostose der hinteren spongiosirten Wand des Pelvis ovalis. 